\title{
A DISCRETE NETWORK APPROXIMATION FOR EFFECTIVE CONDUCTIVITY OF NON-OHMIC HIGH-CONTRAST COMPOSITES*
}

\author{
ALEXEI NOVIKOV ${ }^{\dagger}$
}

\begin{abstract}
We develop a discrete network approximation to effective conductivity of high contrast, highly packed particulate composites with nonlinear constituent relations. The key tool is the perforated medium approach, which provides a simple mathematical justification of the discrete network approximation by variational techniques.
\end{abstract}

Key words. Effective conductivity, discrete network, variational bounds.

AMS subject classifications. 74Q05, 35Q72, 94C05.

\section{Introduction}

The understanding of the overall properties of particulate media is relevant to aspects of virtually every branch of science and engineering, especially materials science, chemical engineering, biology, bioengineering, environmental science, geophysics, and fluid dynamics. The overall properties of high-contrast particulate media are difficult to calculate analytically and numerically. When the concentration of inclusions in the hosting medium (matrix) is high, these properties can be approximated by analogous properties of a discrete network [1-6, 13, 16, 19, 28].

The discrete network approximation is useful because it is easy to implement numerically, and at the same time it captures geometric patterns of the location of inclusions in the matrix. The importance of geometric patterns in the evaluation of the overall properties of high contrast particulate media can already be seen for periodic composites. At moderate volume fraction $\hat{a}$, the effective conductivity is of the order of the conductivity of the matrix (see, for example, [23, 24]). If, however, the contrast ratio of the constituents is assumed to be $\infty$, then for the same volume fraction of disks (equal to $\pi / 4$ ) for the hexagonal lattice, the effective conductivity $\hat{a}=O(1)[6]$, while for the square lattice $\hat{a}=\infty[18]$.

The first rigorous mathematical justification of a network approximation was developed in [7-10] for high-contrast media that arises in imaging. For particulate composites and suspensions with linear constitutive relations, a network approximation was developed in [2-6]. The main objective of this paper is to develop and justify a discrete network for particulate composites where the hosting medium has a nonlinear constitutive relation. The main motivation of this work is to explain a perforated medium approach, a mathematical tool that allows to construct discrete network approximations to effective properties of high-contrast materials. This approach allows a simple derivation and justification of a discrete network approximation.

1.1. Formulation. Consider a two-dimensional model of a two-phase composite that consists of a matrix in which a large number of perfectly conducting inclusions are randomly distributed. The composite is modeled by a rectangle $\Pi=$ $[-1,1] \times[-L, L]$. The inclusions are modeled by identical non-overlapping disks $B_{i}$

*Received: April 11, 2009; accepted (in revised version): June 26, 2009. Communicated by Jack Xin.

${ }^{\dagger}$ Department of Mathematics, McAllister Building, Pennsylvania State University, University Park, PA 16802, USA (anovikov@math.psu.edu). 
of radius $R, i=1, \ldots N$, where $N$ is the number of inclusions, see figure 1.1. The concentration of the inclusions is high, that is, the characteristic distance between two neighboring inclusions is much smaller than their radius. Then the domain

$$
Q=\Pi \backslash \bigcup_{i=1}^{N} B_{i}
$$

models the matrix of the composite.

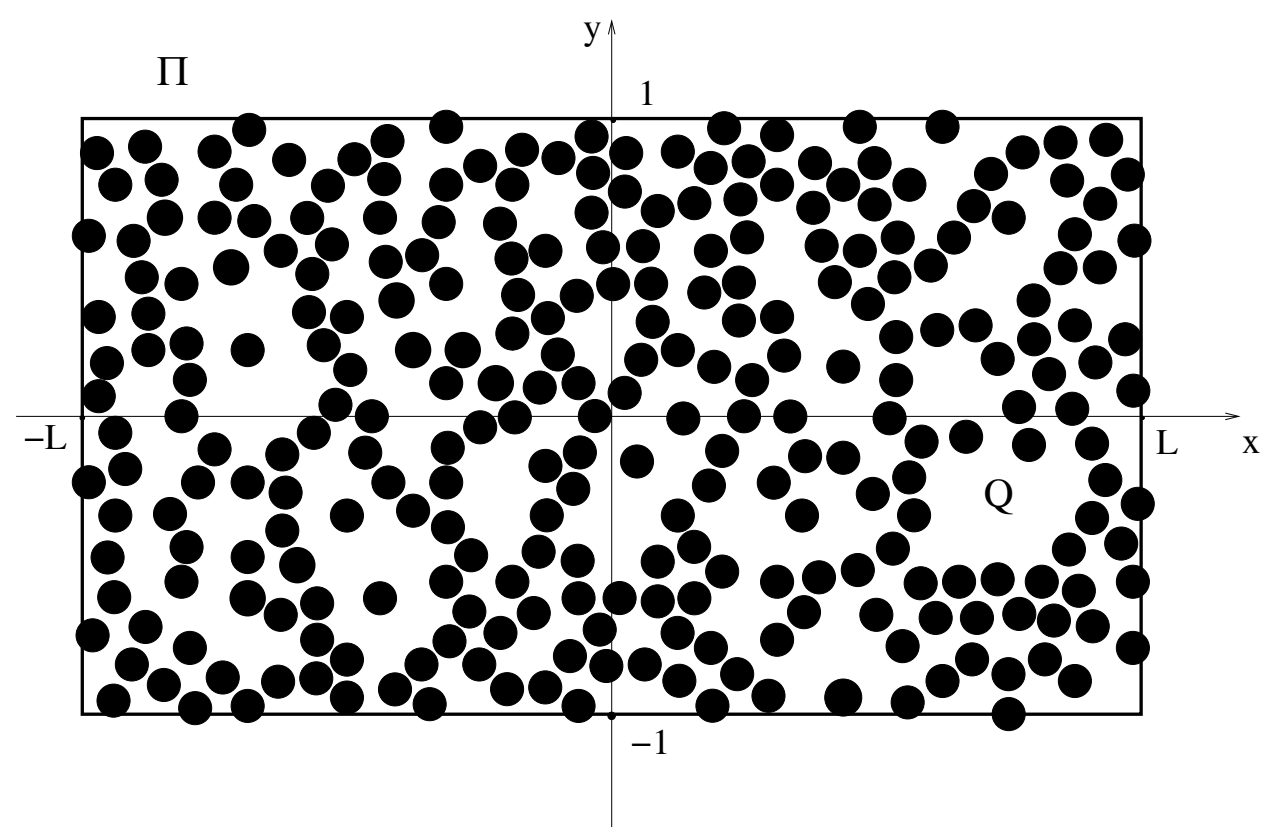

FIG. 1.1. Matrix with inclusions

The matrix is a (nonlinear) homogeneous medium, and we will assume that the current-electric field relation is the power law:

$$
\boldsymbol{J}=|\boldsymbol{E}|^{p-2} \boldsymbol{E}, p \geq 2, p \in \mathbb{N} \text {. }
$$

The electric field is determined by a potential $\boldsymbol{E}=-\nabla \phi(x, y)$, and the current field is divergence free, $\nabla \cdot \boldsymbol{J}=0$. So in the matrix the electric potential satisfies

$$
\nabla \cdot|\nabla \phi|^{p-2} \nabla \phi=0
$$

The inclusions are assumed to be ideally conducting:

$$
\nabla \phi(x, y)=0, \text { on } B_{i}, i=1, \ldots, N \text {. }
$$

There are no sinks or sources inside the disks, which amounts to

$$
\int_{\partial B_{i}} \boldsymbol{n} \cdot \boldsymbol{J} d s=\int_{\partial B_{i}}|\nabla \phi|^{p-2} \boldsymbol{n} \cdot \nabla \phi d s=0
$$


As it is natural, when bulk effective properties are discussed, we impose the constant applied field boundary conditions (Dirichlet boundary conditions on the top and the bottom boundaries and Neumann boundary conditions on lateral boundaries):

$$
\text { a) } \phi(x, \pm 1)= \pm 1, b) \boldsymbol{n} \cdot \nabla \phi( \pm L, y)=0 .
$$

The effective conductivity is

$$
\hat{a}=\frac{1}{2|Y|} \int_{Q} \boldsymbol{J} \cdot \boldsymbol{E} d x d y=\frac{1}{8 L} \int_{Q}|\nabla \phi|^{p} d x d y .
$$

We will ignore the normalizing constant $8 L$ and set

$$
\hat{a}=\int_{Q}|\nabla \phi|^{p} d x d y
$$

1.2. Discrete network approximation. Our goal is to investigate $\widehat{a}$ in (1.7) when the concentration of inclusions is high, and their geometric distribution is far from periodic. For general composite materials homogenization, variational bounds and other methods are used (see e.g. [25] for a good review of extensive literature on the subject). These methods, however, do not provide an accurate description of the effective properties of high-contrast particulate materials at high concentration of particles, because such properties depend on singularities of the solutions, e.g., equations (1.3)-(1.7). These singularities occur exactly in the areas (necks) between almost touching particles (see figure 1.3). The location of these singularities can be characterized naturally by the location of edges in the discrete network. Therefore networks, where vertices are the particles, naturally arise. Asymptotic discrete network approximations on these graphs are then used to characterize these properties in such materials (see, e.g., $[1,13,16,19,28]$ ). The effective conductivity $\hat{a}(1.7)$ or, more generally, other bulk properties of a composite are then determined approximately by the properties of this network. This representation by a network shows clearly geometric patterns in a particulate medium, for example clusters of particles.

The discrete network approximation is a discrete network, which models the composite, and a discrete energy, a function that approximates the value of the effective conductivity. Here we give a conceptual description of the discrete network approximation, and review the rigorous construction in section 2 .

The discrete network is the Delaunay triangulation, associated with the centers of the inclusions: the vertices are centers of the inclusions and the edges are the edges of the Delaunay triangulation, as depicted on figure 1.2. In addition to the $N$ vertices which correspond to inclusions we assign the vertex $\mathrm{x}_{N+1}$ to the whole upper boundary and $\mathrm{x}_{N+2}$ to the whole lower boundary, respectively. In order to define the discrete energy we assign a potential ${ }^{1} t_{i}$ to each vertex $\mathrm{x}_{i}$ and assign the local energy $g_{i j}=g_{i j}\left(\Pi_{i j}, t_{i}, t_{j}\right)$ to each edge $\Pi_{i j}$. Then the discrete energy is

$$
\widehat{a}_{d}=\min _{t_{i}, i=1, \ldots, N} \sum_{\Pi_{i j}} g_{i j}\left|t_{i}-t_{j}\right|^{p}, t_{N+1}=1, t_{N+2}=-1,
$$

where the summation is over all edges $\Pi_{i j}$.

\footnotetext{
${ }^{1}$ Note that condition (1.4) implies that $\phi$ is a constant on each inclusion.
} 


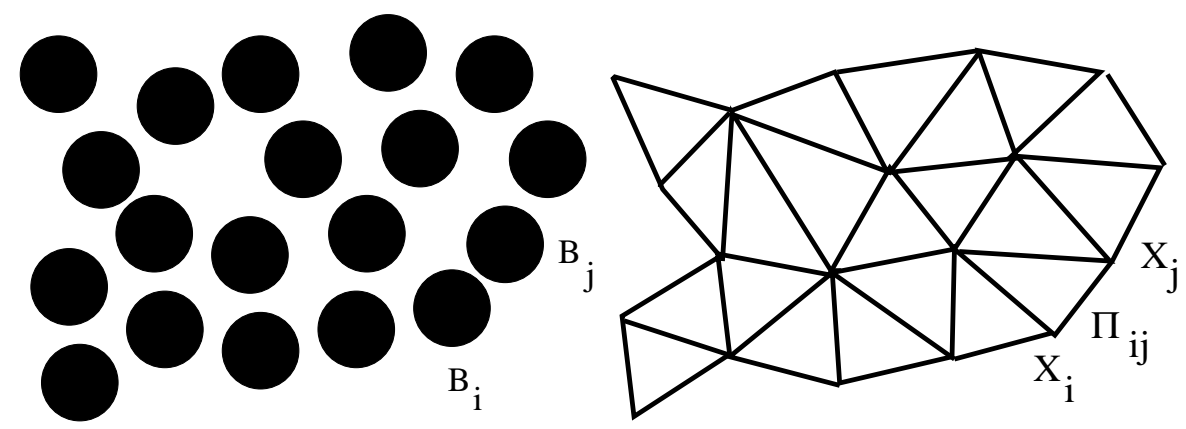

FIG. 1.2. Particulate composite and its network

1.3. Approximation theorems. Rigorous mathematical justification of discrete network approximation is based on geometric and asymptotic arguments which are coupled together. As a result, all rigorous results [2-10] on construction of asymptotic discrete network approximations for high-contrast composites are complicated and produced on a case by case basis. Such complicated constructions are not attractive for practitioners. The purpose of this paper is to address this issue by separating the geometric and the asymptotic arguments.

The geometric argument says that a good approximation to effective conductivity arises if only take into account nearest neighbor pairwise interactions. Mathematically it means that it suffices to take into account only interaction of inclusions that are connected by an edge in the Delaunay triangulation, as it is done in (1.8). Our first theorem shows the error of such approximation.

THEOREM 1.1. (Pairwise interaction approximation) The weights $g_{i j}$ in (1.8) could be chosen so that the effective conductivity $\widehat{a}$ (1.7) could be approximated by the discrete energy $\widehat{a}_{d}(1.8)$ in the sense that

$$
\min _{t_{i}, i=1, \ldots, N} \sum_{\Pi_{i j}} g_{i j}\left|t_{i}-t_{j}\right|^{p} \leq \hat{a} \leq \min _{t_{i}, i=1, \ldots, N} \sum_{\Pi_{i j}}\left(g_{i j}+C\right)\left|t_{i}-t_{j}\right|^{p}
$$

where $t_{N+1}=1, t_{N+2}=-1$, and the constant $C=C(R, L)$ depends on the radius of the inclusions $R$, and the size of the composite $L$.

Theorem 1.1 follows from variational upper and lower bounds obtained in Sections 2.2 and 2.3 respectively. The theorem says that if we take into account only the effect of pairwise interaction between inclusions, then we make at most $O(1)$ error. Therefore, this theorem is ineffective, if the effective conductivity $\widehat{a}$ is itself $O(1)$. The effective conductivity, however, becomes large when the concentration of inclusions is high, because the weights $g_{i j}$ in (1.9) tend to $\infty$ as the interparticle distance between the inclusions tends to zero (for a pair of inclusions $B_{i}$ and $B_{j}$, connected by the edge $\Pi_{i j}$, the interparticle distance is the distance between their centers: $\left.\delta_{i j}=\left|\mathrm{x}_{i}-\mathrm{x}_{j}\right|-2 R\right)$. The next lemma provides an explicit asymptotic behavior of $g_{i j}$.

Lemma 1.2. (Asymptotics of $g_{i j}$ ) Suppose the weights $g_{i j}$ are chosen as in Theorem 1.1. Consider any two closely spaced inclusions $B_{i}$ and $B_{j}$, as depicted on figure 


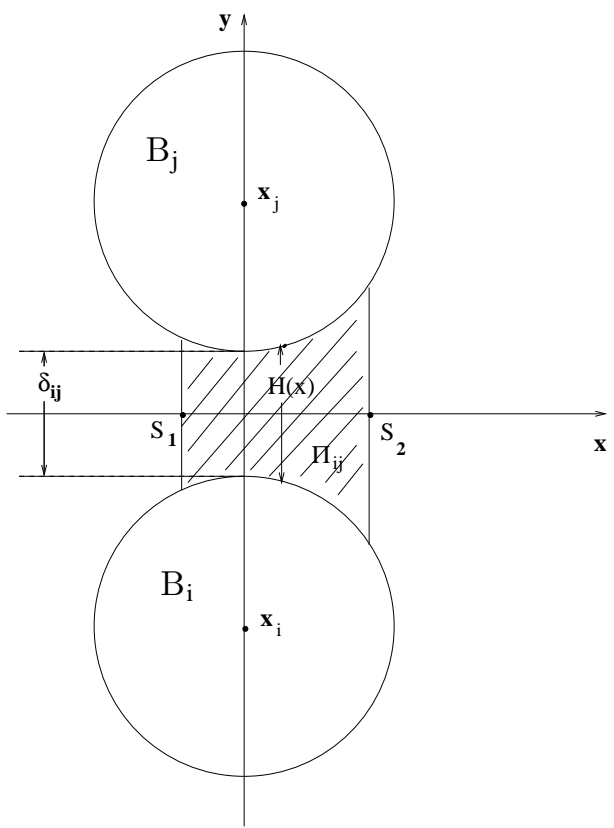

FIG. 1.3. The hatched region is the neck between two neighbors

1.3. Suppose the interparticle distance between them is $\delta_{i j}$. Then

$$
\frac{g_{i j}-g_{i j}^{o}}{g_{i j}^{o}} \rightarrow 0, \text { as } \delta_{i j} \rightarrow 0,
$$

where the constants

$$
g_{i j}^{o}=\mathbf{G}\left(\delta_{i j}\right)
$$

are given in the following table

\begin{tabular}{|c|c|c|}
\hline & $d=2$ & $d=3$ \\
\hline$p=2$ & $\pi\left(\frac{R}{\delta_{i j}}\right)^{1 / 2}$ & $\pi R \ln \left(\frac{R}{\delta_{i j}}\right)$ \\
\hline$p=3$ & $\frac{\pi}{2 R}\left(\frac{R}{\delta_{i j}}\right)^{3 / 2}$ & $\frac{\pi}{2}\left(\frac{R}{\delta_{i j}}\right)$ \\
\hline$p=4$ & $\frac{3 \pi}{8 R^{2}}\left(\frac{R}{\delta_{i j}}\right)^{5 / 2}$ & $\frac{\pi}{8 R}\left(\frac{R}{\delta_{i j}}\right)^{2}$ \\
\hline general & $\frac{\pi a_{p}}{R^{p-2}}\left(\frac{R}{\delta_{i j}}\right)^{p-3 / 2}$ & $\frac{\pi}{2^{p-1}(p-2) R^{p-3}}\left(\frac{R}{\delta_{i j}}\right)^{p-2}$ \\
\hline
\end{tabular}

and $a_{p}$ are the coefficients of the Taylor series

$$
\frac{1}{\sqrt{1-x}}=1+\frac{x}{2}+\frac{3 x^{2}}{8}+\cdots+a_{p} x^{p-2}+\ldots
$$

For a neck $\Pi_{i j}$ between an inclusion $B_{i}$ and the flat boundary $j=N$ or $j=N+1$. $g_{i j}^{o}$ is given by

$$
g_{i j}^{o}=2^{p-1} \mathbf{G}\left(2 \delta_{i j}\right) .
$$


The asymptotic argument relies on Lemma 1.2, and it allows us to conclude that if all interparticle distances are sufficiently small, then $\hat{a}_{d}$ approximates $\hat{a}$, as described in the following theorem.

ThEOREM 1.3. Suppose the dimension $d=2$ or $d=3$. Consider a high-contrast power-law composite. Suppose for all $\Pi_{i j}$ we have that $\delta_{i j} \leq \delta$, and the weights $g_{i j}$ are chosen as in the Theorem 1.1. Then for $p \geq d+1$

$$
\frac{\left|\hat{a}-\hat{a}_{d}\right|}{\hat{a}_{d}} \leq C \delta^{p-3 / 2}
$$

where the constant $C_{1}$ may depend on $R$ and $L$ only; for $p=d=3$

$$
\frac{\left|\hat{a}-\hat{a}_{d}\right|}{\hat{a}_{d}} \leq C \delta
$$

for $p=d=2$

$$
\frac{\left|\hat{a}-\hat{a}_{d}\right|}{\hat{a}_{d}} \leq C \sqrt{\delta}
$$

for $p=2, d=3$

$$
\frac{\left|\hat{a}-\hat{a}_{d}\right|}{\hat{a}_{d}} \leq \frac{C}{|\ln \delta|}
$$

A few remarks are in order about Theorem 1.3. Firstly, the result estimates the relative error of two asymptotically large quantities: the effective conductivity and the energy of the discrete network. The latter is a simple polynomial. Its evaluation is simpler, especially in three dimensions, than the evaluation of (1.7). This fact is the main attractive feature of the discrete network approximation. Secondly, the discrete network provides an approximation. The usefulness of this approximation should be determined by the problem at hand: if computational complexity is an issue, but the accuracy is less important, then the discrete network approximation is a good choice for such computation; if, on the other hand, computational complexity is low, then other methods, e.g. finite elements, might be more attractive. Thirdly, for composites with a nonlinear constitutive relation, the constants $g_{i j}$ may depend on the value of $t_{i}$ and $t_{j}$. In our case $g_{i j}$ can be chosen independent of $t_{i}$. Fourthly, it turns out that the formulas in table 1.11 depend on the (possibly singular) curvature of the inclusions in the necks. It implies that the analysis, presented in this paper, is directly applicable to the case when inclusions are ellipses or rectangles. Finally, the main advantage of the discrete network approximation can be seen when the concentration of inclusions is high, for moderate concentrations discrete network approximation should be used together with other methods of characterization of effective properties of composites.

Also note that the explicit asymptotic formulas for $g_{i j}$ are not computed in this work. In table (1.11) we give $g_{i j}^{o}$, which are only the leading asymptotic terms of $g_{i j}$, as $\delta_{i j} \rightarrow 0$. Calculation of other singular terms in the asymptotic expansion of $g_{i j}$ could be done as in [4]. If we use $g_{i j}^{o}$ from table (1.11) instead of $g_{i j}$ in the functional (1.8), then with such choice we will obtain worse error estimates, that are summarized in the next Corollary. 
Corollary 1.4. Suppose the dimension $d=2$ or $d=3$. Consider a high-contrast power-law composite. Suppose for all $\Pi_{i j}$ we have that $\delta_{i j} \leq \delta$. Let

$$
\hat{a}_{d}^{o}=\min _{t_{i}, i=1, \ldots, N} \sum_{\Pi_{i j}} g_{i j}^{o}\left|t_{i}-t_{j}\right|^{p},
$$

where the weights $g_{i j}^{o}$ are chosen as in the Lemma 1.2. Then for $p \geq d+1$,

$$
\frac{\left|\hat{a}-\hat{a}_{d}^{o}\right|}{\hat{a}_{d}^{o}} \leq C \delta,
$$

where the constant $C$ may depend on $R$ and $L$ only; for $p=d=3$

$$
\frac{\left|\hat{a}-\hat{a}_{d}^{o}\right|}{\hat{a}_{d}^{o}} \leq C \delta|\ln \delta|
$$

for $p=d=2$

$$
\frac{\left|\hat{a}-\hat{a}_{d}^{o}\right|}{\hat{a}_{d}^{o}} \leq C \sqrt{\delta}
$$

for $p=2, d=3$

$$
\frac{\left|\hat{a}-\hat{a}_{d}^{o}\right|}{\hat{a}_{d}^{o}} \leq \frac{C}{|\ln \delta|}
$$

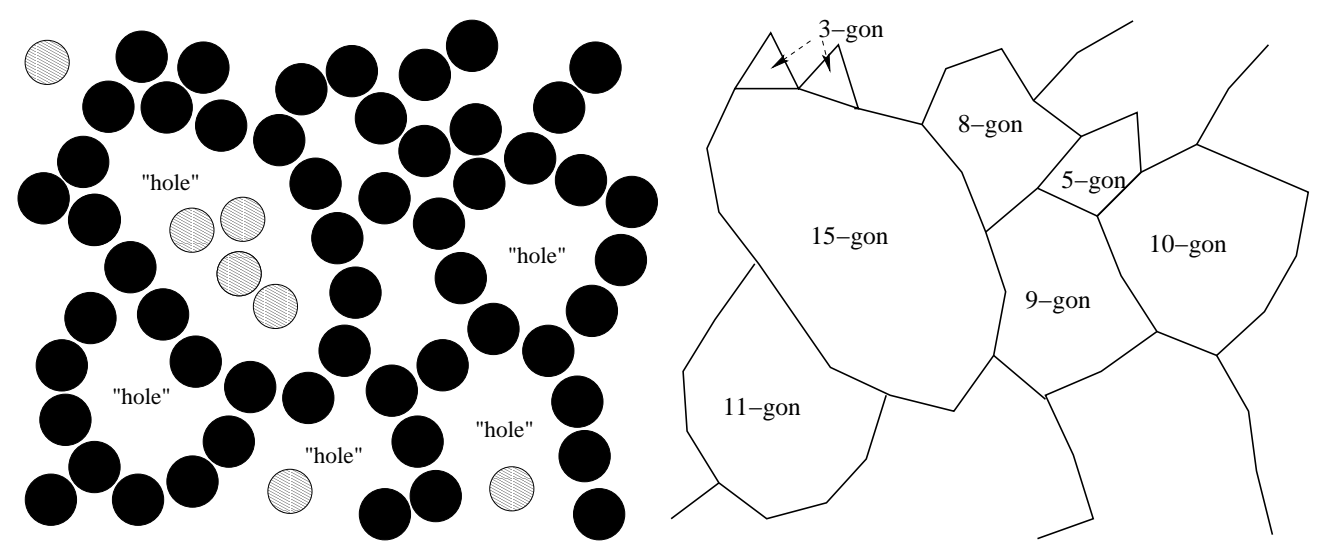

FIG. 1.4. The conducting cluster in a composite with "holes".

Further corollaries from Theorem 1.3 could be obtained, when we modify the assumption that all $\delta_{i j} \leq \delta, \delta \ll 1$. This is the so-called close packing condition [5]. Composites that satisfy this condition are such that the inclusions form a perturbed hexagonal close packing (cubic or hexagonal close packing in three dimensions). Hence the previous theorem and the corollary cover only these composites. It is expected, however, that the discrete network approximation is applicable to a wider range of distributions of inclusions in a composite. Thus this assumption is restrictive and it is desirable to relax it. A possible approach was suggested in $[3,6]$, where we introduced 
the $\delta-\mathbf{N}$ close packing condition, which loosely speaking allows for "holes" with the perimeter of order $\mathbf{N} R$ in the conducting spanning cluster (see Fig. 1.4). Such a generalization, however, cannot easily be generalized to three dimensions [3]. Our proof of Theorem 1.3 allows us to avoid working with $\delta-\mathbf{N}$ close packing condition and obtain a result, which is more general, it is applicable in three dimensions, and, at the same time, it has a natural physical interpretation. The result is stated in the next theorem, which we precede with the following definition.

DEFINITION 1.5. We say that a discrete network with vertices $x_{i}, i=1, \ldots, N$, contains a $\delta$-percolation cluster, if there is a sequence of vertices connecting the top and the bottom boundary of the composite, such that their consecutive interparticle distances are less than $\delta$. More specifically, there exist vertices

$$
\mathrm{x}_{1}, \mathrm{x}_{2}, \ldots, \mathrm{x}_{k}, \text { such that }{ }^{2} \delta_{N+1,1} \leq \delta, \delta_{k, N+2} \leq \delta \text {, and } \delta_{i, i+1} \leq \delta, i=1,2, \ldots, k-1 .
$$

THEOREM 1.6. Theorem 1.3 and Corollary 1.4 hold for discrete networks that contain $\delta$-percolation clusters.

The main objective of this work is to show how to separate geometric and asymptotic arguments in the construction of the discrete network approximation. This objective dictated the organization of the paper. Section 2 is the main part of the paper. We develop there a geometric theory of discrete networks, which is independent of any asymptotic approximations. In particular, we prove there Theorem 1.1, which, in contrast to previous works, does not require any asymptotic analysis. In section 3 we present the asymptotic theory of discrete networks by proving Lemma 1.2 and Theorem 1.3. In section 4 we prove Theorem 1.6 only, since Corollary 1.4 is its particular case.

\section{Perforated medium approach}

We explain here how a discrete network arises for (1.3)-(1.7) using the idea of a perforated medium.

The key observation was made for a periodic matrix with linear constitutive relation by J.B. Keller in [18] $(p=2$ in (1.3)): the dominant contribution to the effective conductivity $\hat{a}$ comes from areas (necks) between closely spaced disks (see shaded regions on figure $2.1(\mathrm{~b})$ ). As it follows from the asymptotic analysis in this paper, the same observation holds true for power-law media with $p \geq 1.5$ in two dimensions and $p \geq 2$ in three dimensions. Since $p \geq 2$ in (1.2), the observation is valid in our set-up:

$$
\hat{a}=\int_{Q}|\nabla \phi|^{p} d x d y \approx \sum_{\Pi_{i j}} \int_{\Pi_{i j}}|\nabla \phi|^{p} d x d y,
$$

where $\Pi_{i j}$ is the neck between the $i$ th and $j$ th disk (see figure 2.1(b)).

We can interpret the approximation (2.1) as an equivalence relation between effective properties of two composites - the original one and the one perforated by triangular holes. Physically it means the following. Imagine that the matrix occupies only necks, and in the triangles we have voids. Then the effective conductivity of the original composite is almost the same as the effective conductivity of a composite perforated by triangular voids. Such a point of view is proven to be useful in analysis

\footnotetext{
${ }^{2}$ Recall that the bottom boundary is indexed by $N+1$, and the top boundary is indexed by $N+2$.
} 
of diffusion at high Péclet numbers in stationary two-dimensional cell-like flows [27] and the rate of viscous dissipation of a two-dimensional suspension of rigid particles in an incompressible Stokesian fluid [4]. We call this matrix with triangular holes a perforated medium:

$$
\Pi_{o}=Q \backslash \bigcup_{i, j, k} \Delta_{i k j}=\bigcup_{i, j} \Pi_{i j}
$$

A rigorous construction of the triangular holes is based on the Voronoi tessellation and it is summarized as follows (see also [6] and [3] for two- and three-dimensional cases, respectively).

Consider the Voronoi tessellation associated with the centers of the inclusions. If two Voronoi cells with centers at $\mathrm{x}_{i}$ and $\mathrm{x}_{j}$ share a common edge (face, in 3D), then they are neighbors, and we connect them with an edge $\Pi_{i j}$; see figure 2.1.

By connecting the centers of inclusions with the corresponding vertices of its Voronoi cell, we can find the vertices of triangles where the connecting lines intersect boundaries of inclusions. This construction uniquely determines (up to some degeneracies) the triangle-neck partition as on figure 2.1(b), and therefore the perforated domain. A more detailed description can be found in [6].

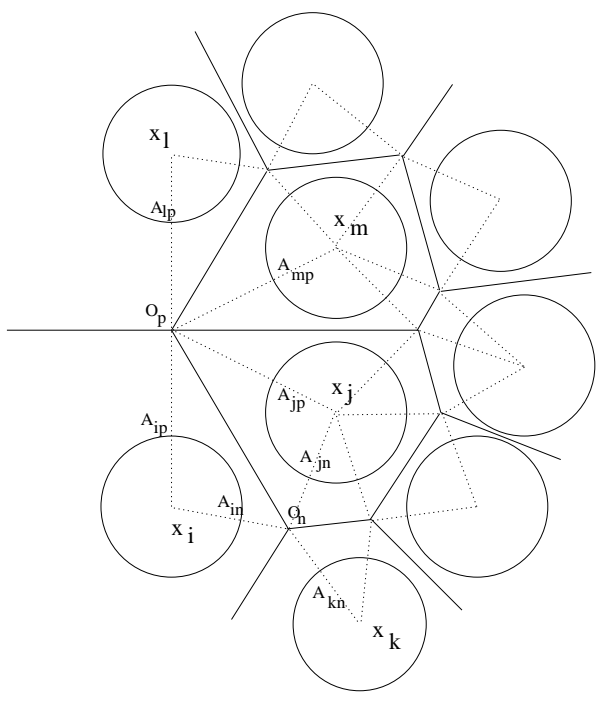

(a) Voronoi tessellation

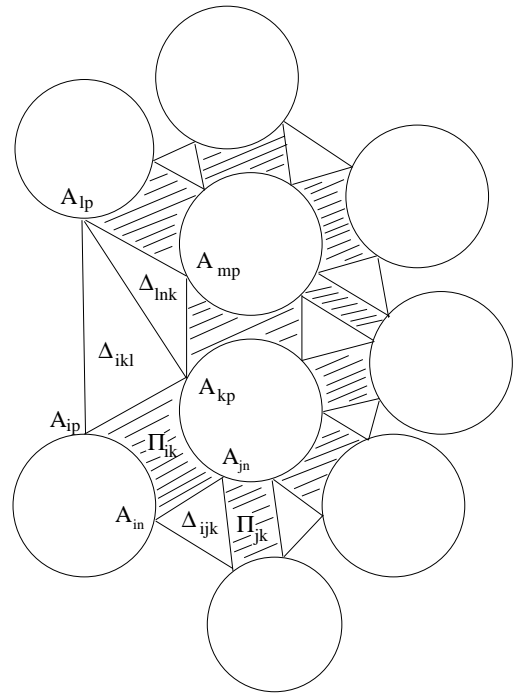

(b) Triangle-neck partition

FIG. 2.1. Perforated medium comprises inclusions and necks

The idea of a perforated medium is attractive, because it makes the appearance of discrete networks intuitively clear. It also is very useful in the construction of variational bounds. These bounds are based on variational principles, that we review next.

2.1. Variational Principles. It is well-known [17] that the problem (1.3)(1.7) admits a variational formulation as follows. Let the potential $\phi(x, y)$, be a function from the functional space

$$
V=\left\{\phi \in W^{1, p}(Q): \nabla \phi(x, y)=0 \text { on } \partial B_{i} ; \phi(x, \pm 1)= \pm 1\right\},
$$


and define an energy functional

$$
I[\phi]=\int_{Q}|\nabla \phi|^{p} d x d y, \phi \in V
$$

Then the effective conductivity (1.7) is the minimum of this energy functional

$$
\hat{a}=\min _{\widetilde{\phi} \in V} I[\widetilde{\phi}],
$$

moreover, the minimizer $\phi$ of (2.5) satisfies (1.4), (1.6) a) because $\phi \in V$, and (1.3), $(1.5),(1.6) \mathrm{b})$ because these are the Euler-Lagrange equations of the variational formulation (2.5).

Using the Legendre transform, it is possible [17] to represent the effective conductivity as the maximum value of the dual functional.

$$
I^{*}[u]=\left[p \int_{y= \pm 1}|\boldsymbol{u}|^{p-2} \boldsymbol{n} \cdot \boldsymbol{u} d s-(p-1) \int_{Q}|\boldsymbol{u}|^{p} d x d y\right], \quad \boldsymbol{u} \in V^{*},
$$

where $d s= \pm d x$ for $y= \pm 1$, and the dual space

$$
V^{*}=\left\{\boldsymbol{u} \in L^{p}\left(Q, \mathbb{R}^{2}\right): \nabla \cdot|\boldsymbol{u}|^{p-2} \boldsymbol{u}=0, \int_{\partial B_{i}}|\boldsymbol{u}|^{p-2} \boldsymbol{n} \cdot \boldsymbol{u} d s=0, \boldsymbol{n} \cdot \boldsymbol{u}( \pm L, y)=0\right\}
$$

Above $L^{p}\left(Q, \mathbb{R}^{2}\right)=L^{p}(Q)^{2}$ denotes the space of vector valued functions $\boldsymbol{u}=\left(u_{1}, u_{2}\right)$, such that $u_{1}(x, y) \in L^{p}(Q)$ and $u_{2}(x, y) \in L^{p}(Q)$.

Then the effective conductivity (1.7) is the maximum of this dual energy functional:

$$
\hat{a}=\max _{\widetilde{\boldsymbol{u}} \in V^{*}} I^{*}[\widetilde{\boldsymbol{u}}],
$$

moreover, the maximizer of (2.8) satisfies $\boldsymbol{u}=\nabla \phi$, where $\phi$ solves (1.3)-(1.6).

The main use of the variational formulation here (as well as in other problems in Material Science, see [12,25] for extensive references; and in Fluid Dynamics [26, 27]) is to provide upper and lower estimates for the effective conductivity by choosing appropriate trial functions from $V$ and $V^{*}$, respectively. Note that, as it is typical in calculus of variations, the dual variational formulation $(2.6),(2.7)$ is more complicated than the original one (2.4), (2.3) mainly because the space $V^{*}$ is characterized by constraints, which are more difficult to handle. As a result 'good' lower bounds are much harder to obtain. A notion of a perforated medium, that we described above is designed specifically to overcome this difficulty.

2.2. Variational lower bound. Since $\Pi_{o} \subset Q$, then the effective conductivity of the perforated domain does not exceed the effective conductivity of the whole domain

$$
\min _{\phi \in V_{o}} \int_{\Pi_{o}}|\nabla \phi|^{p} d x d y \leq \hat{a}=\min _{\phi \in V} \int_{Q}|\nabla \phi|^{p} d x d y
$$

where the space $V$ is defined in (2.3) and

$$
V_{o}=\left\{\phi \in W^{1, p}\left(\Pi_{o}\right): \phi(x, y)=t_{i} \text { on } \partial B_{i}, \phi(x, \pm 1)= \pm 1\right\} .
$$


A significant advantage of the minimization over a perforated domain is that the global minimization problem over $\Pi_{o}$ can be split into two consecutive problems: one of them is on a single neck $\Pi_{i j}$, and the other one is a minimization problem of discrete variables $t_{i}$. This almost trivial observation is the key step in construction of effective lower bounds for the effective conductivity, and we give this observation in more detail in the next lemma.

LEMma 2.1. (Iterative minimization lemma). Consider a high-contrast power-law composite. Let $\Pi_{o}$ be its perforated domain. Then

$$
\min _{\phi \in V_{o}} \int_{\Pi_{o}}|\nabla \phi|^{p} d x d y=\min _{t_{i}, i=1, \ldots, N} \sum_{\Pi_{i j}} g_{i j}\left|t_{i}-t_{j}\right|^{p}, \text { with } t_{N+1}=1, t_{N+2}=-1 \text {, }
$$

where

$$
g_{i j}=\min _{\phi} \int_{\Pi_{i j}}|\nabla \phi|^{p} d x d y, \phi(x, y) \in V_{\Pi_{i j}}
$$

where the space

$$
V_{\Pi_{i j}}=\left\{\phi \in W^{1, p}\left(\Pi_{i j}\right):\left.\phi(x, y)\right|_{\partial B_{i}}=1 / 2,\left.\phi(x, y)\right|_{\partial B_{j}}=-1 / 2\right\}
$$

Moreover the minimizer $\phi$ of (2.12) satisfies

$$
\begin{aligned}
\nabla \cdot|\nabla \phi|^{p-2} \nabla \phi & =0, \quad \text { for }(x, y) \in \Pi_{i j}, \\
\left.n \cdot \nabla \phi(x, y)\right|_{\partial \Pi_{i j}^{ \pm}} & =0,
\end{aligned}
$$

where $\partial \Pi_{i j}^{ \pm}$are "lateral" boundaries of a neck as depicted on figure 2.2.

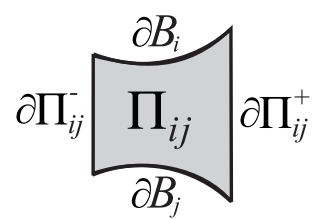

FIG. 2.2. Neck $\Pi_{i j}$ and its boundary.

The proof of this lemma is elementary. It is given in [4] for a network model of suspensions in an incompressible fluid, that satisfies a (linear) Stokes law. In the general nonlinear case the coefficients $g_{i j}$ may depend on $t_{i}$ and $t_{j}$. For a power-law medium they do not depend on $t_{i}$ and $t_{j}$, because if $\phi$ is the solution to (2.14), then $\left(t_{i}-t_{j}\right) \phi+\left(t_{i}+t_{j}\right) / 2$ is the solution to

$$
\begin{aligned}
\nabla \cdot|\nabla \phi|^{p-2} \nabla \phi & =0, \quad \text { for }(x, y) \in \Pi_{i j}, \\
\left.\phi(x, y)\right|_{\partial B_{i}} & =t_{i}, \\
\left.\phi(x, y)\right|_{\partial B_{j}} & =t_{j}, \\
\left.n \cdot \nabla \phi(x, y)\right|_{\Pi_{i j}^{ \pm}} & =0 .
\end{aligned}
$$


A lower bound on $g_{i j}$ in (2.12) can be obtained using variational duality ${ }^{3}$ :

$$
g_{i j}=\max _{\boldsymbol{u} \in V_{\Pi_{i j}}^{*}}\left[\frac{p}{2} \int_{\partial B_{i} \cup \partial B_{j}}|\boldsymbol{u}|^{p-2} \boldsymbol{n} \cdot \boldsymbol{u} d s-(p-1) \int_{\Pi_{i j}}|\boldsymbol{u}|^{p} d x d y\right],
$$

where the space

$$
V_{\Pi_{i j}}^{*}=\left\{\boldsymbol{u} \in L^{p}\left(\Pi_{i j}, \mathbb{R}^{2}\right): \nabla \cdot|\boldsymbol{u}|^{p-2} \boldsymbol{u}=0, \boldsymbol{n} \cdot \boldsymbol{u}=0 \text { on } \partial \Pi_{i j}^{ \pm}\right\} .
$$

Compare the spaces $V^{*}(2.7)$ and $V_{\Pi_{i j}}^{*}(2.17)$ and observe that the complicated integral condition

$$
\int_{\partial B_{i}}|\boldsymbol{u}|^{p-2} \boldsymbol{n} \cdot \boldsymbol{u} d s=0
$$

in (2.7) has no analog in (2.17). This simplification is raison d'être of the perforated medium. In our scalar case the simplification does not look so drastic, in vectorial problems it is significant, see [4]. We conclude that Lemma 2.1 and inequality (2.9) imply a lower bound on the effective conductivity:

$$
\hat{a} \geq \min _{t_{i}, i=1, \ldots, N} \sum_{\Pi_{i j}} g_{i j}\left|t_{i}-t_{j}\right|^{p}, \text { with } t_{N+1}=1, t_{N+2}=-1,
$$

where $g_{i j}$ are given by (2.12).

2.3. Variational upper bound. The perforated medium approach provides a natural variational lower bound, as described above. It does not, however, provide an upper bound. In order to obtain such an upper bound, all previous works [2-10] explicitly constructed trial fields for the direct variational formulation (2.4), (2.5) on the whole domain $Q$. These trial fields used the assumption that the inclusions are closely spaced. As a consequence, the geometric step, and the asymptotic step of the construction of the discrete network were not separated. Our next lemma allows to do this separation.

Lemma 2.2. As in Lemma (2.1) we denote

$$
g_{i j}=\min _{\phi} \int_{\Pi_{i j}}|\nabla \phi|^{p} d x d y, \phi(x, y) \in V_{\Pi_{i j}},
$$

then there is a constant $C=C(R, L)$, that depends on the radius of the inclusions $R$, and the size of the composite $L$, such that we have an upper bound

$$
\hat{a} \leq \min _{t_{i}, i=1, \ldots, N} \sum_{\Pi_{i j}}\left(g_{i j}+C\right)\left|t_{i}-t_{j}\right|^{p}, \text { with } t_{N+1}=1, t_{N+2}=-1 .
$$

Proof. Let $\phi_{i j}^{\text {neck }} \in V_{\Pi_{i j}}$ (2.13) be the minimizer of the energy integral (2.12) on the neck $\Pi_{i j}$ described in Lemma 2.11. As we show in the next subsection 2.4, we can obtain a crucial estimate

$$
\left|\nabla \phi_{i j}^{\text {neck }}\right| \leq C, \text { for }(x, y) \in \partial \Pi_{i j}
$$

where the constant $C=C(R)$ depends on the radius of inclusions $R$ only. Fix

\footnotetext{
${ }^{3}$ There is a factor of 2 in (2.16), compared to (2.6), because $\phi= \pm 1 / 2$ on the Dirichlet boundary (inclusions), whereas $\phi= \pm 1$ on the corresponding Dirichlet boundary (upper and lower boundaries) in $(2.3)$.
} 


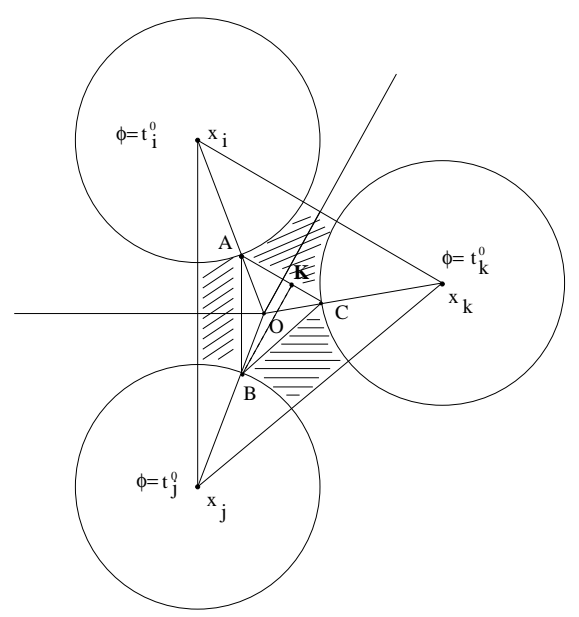

FIG. 2.3. Typical $\triangle A B C \equiv \Delta_{i j k}$, half-necks are hatched.

an arbitrary $t_{i}, i=1,2, \ldots, N$, and for these $t_{i}$ define a function $\phi_{o}(x, y)$ on the perforated domain $\Pi$ as follows:

$$
\phi_{o}(x, y)=\left\{\begin{array}{l}
\left(t_{i}-t_{j}\right) \phi_{i j}^{\text {neck }}(x, y)+\left(t_{i}+t_{j}\right) / 2, \text { if }(x, y) \in \Pi_{i j}, \\
t_{i}, \text { if }(x, y) \in B_{i} .
\end{array}\right.
$$

Clearly $\phi_{o} \in V_{o}$, (defined by (2.10)) and

$$
\left|\nabla \phi_{o}\right| \leq C\left|t_{i}-t_{j}\right|, C=C(R) \text { for }(x, y) \in \partial \Pi_{i j} .
$$

By the Kirszbraun theorem (see e.g. [15], p. 202) there is a Lipschitz continuous extension $\phi_{i j k}^{\text {triangle }}$ of $\phi_{o}$ into each triangle $\Delta_{i j k}$ (depicted on figure 2.3) so that

$$
\left|\nabla \phi_{i j k}^{\text {triangle }}(x, y)\right| \leq C \max \left(\left|t_{i}-t_{j}\right|,\left|t_{i}-t_{k}\right|,\left|t_{j}-t_{k}\right|\right),(x, y) \in \Delta_{i j k}, C=C(R) .
$$

Consider a trial field $\tilde{\phi}$ such that

$$
\tilde{\phi}(x, y)=\left\{\begin{array}{l}
\phi_{o}(x, y), \text { if }(x, y) \in \Pi_{o}, \\
\phi_{i j k}^{\text {triangle }}(x, y), \text { if }(x, y) \in \Delta_{i j k} .
\end{array}\right.
$$

By construction $\tilde{\phi} \in V$ (defined by (2.3)), thus using the direct variational principle $(2.4),(2.5)$ on the whole domain $Q$ we obtain

$$
\begin{aligned}
\hat{a} & \leq \sum_{\Pi_{i j}} g_{i j}\left|t_{i}-t_{j}\right|^{p}+C(R) \sum_{\Delta_{i j k}}\left(\left|t_{i}-t_{j}\right|+\left|t_{i}-t_{k}\right|+\left|t_{j}-t_{k}\right|\right)^{p} \operatorname{Area}\left(\Delta_{i j k}\right) \\
& \leq \sum_{\Pi_{i j}}\left(g_{i j}+C\right)\left|t_{i}-t_{j}\right|^{p},
\end{aligned}
$$

where the constant $C=C(R, L)$ depends of the size of the composite $L$, and the radius of the inclusions $R$ only. 
2.4. Uniform Lipschitz estimates at necks' boundaries. Estimates (2.18) and (2.19) imply Theorem 1.1. Therefore, we complete its proof by obtaining the crucial estimate (2.20). We start with a geometric observation. Typically a neck $\Pi_{i j}$ is not symmetric with respect to the line connecting the centers of the disks $B_{i}$ and $B_{j}$. An example of a neck is given on figure 1.3, where we used the local coordinate system when the centers of the both disks lie on the $y$-axis. In this coordinate system the width of the left half-neck is $\left|S_{1}\right|, S_{1}<0$, and the width of the right half-neck is $\left|S_{2}\right|, S_{2}>0$. Note that inequalities $S_{1}<0 S_{2}>0$ are not true in general. We define the (possibly negative) minimal relative half-neck width as

$$
\beta_{i j}=\min \left(-\frac{S_{1}}{R}, \frac{S_{2}}{R}\right),-1<\beta_{i j}<1 .
$$

LEMma 2.3. For any triangle-neck partition, a neck $\Pi_{i j}$ has either non-small $\delta_{i j}$ : $\delta_{i j}>R / 100$, or non-small $\beta_{i j}>1 / 3$.

Proof. Consider the construction of the Delaunay triangulation, and the triangleneck partition, as depicted on figure 2.1. For a pair of vertices $\mathrm{x}_{i}$ and $\mathrm{x}_{j}$ let $\alpha_{i j}$ be the smallest angle opposite to $\mathrm{x}_{i} \mathrm{x}_{j}$ : for each $\mathrm{x}_{i} \mathrm{x}_{j}$ there are two angles opposite to $\mathrm{x}_{i} \mathrm{x}_{j}$; on figure 2.1(a) they are $\angle \mathrm{x}_{i} \mathrm{x}_{l} \mathrm{x}_{j}$ and $\angle \mathrm{x}_{i} \mathrm{x}_{k} \mathrm{x}_{j}$. For definiteness, assume $\alpha_{i j}=\angle \mathrm{x}_{i} \mathrm{x}_{k} \mathrm{x}_{j}$. Note that $\beta_{i j}=\cos \left(\alpha_{i j}\right)=\sin \left(\pi / 2-\alpha_{i j}\right)$. If $\delta_{i j} \leq R / 100$, then we claim that we have an upper bound $\cos \left(\alpha_{i j}\right)>1 / 3$. Indeed, if $\delta_{i j} \leq R / 100$ then we have lower bounds $\left|\mathrm{x}_{k} \mathrm{x}_{j}\right| \geq 200\left|\mathrm{x}_{i} \mathrm{x}_{j}\right| / 201$, and $\left|\mathrm{x}_{k} \mathrm{x}_{i}\right| \geq 200\left|\mathrm{x}_{i} \mathrm{x}_{j}\right| / 201$. Thus $\alpha_{i j}$ could be at most $\pi / 3+\varepsilon$, for some $\varepsilon \ll 1$. The estimate $\beta_{i j}=\cos \left(\alpha_{i j}\right)>1 / 3$ now follows from the law of cosines.

Observe that if a neck $\Pi_{i j}$ has a non-small $\delta_{i j}, \delta_{i j}>R / 100$, then the crucial estimate (2.20) is immediate. Hence we are left to show the following result.

Proposition 2.4. Consider a neck $\Pi_{i j}$ such that its relative half-neck width $\beta_{i j}>$ $1 / 3$, as on figure 2.2. Suppose $\phi$ satisfies

$$
\begin{aligned}
\nabla \cdot|\nabla \phi|^{p-2} \nabla \phi & =0, \quad \text { for }(x, y) \in \Pi_{i j}, \\
\left.n \cdot \nabla \phi(x, y)\right|_{\partial \Pi_{i j}^{ \pm}} & =0, \\
\phi(x, y) & =1 / 2, \quad(x, y) \in \partial B_{i}, \\
\phi(x, y) & =-1 / 2, \quad(x, y) \in \partial B_{j} .
\end{aligned}
$$

Then

$$
|\nabla \phi| \leq C, \text { for }(x, y) \in \partial \Pi_{i j}^{ \pm},
$$

where the constant $C=C(R)$ depends on $R$ only.

Proof. Since $\phi$ satisfies homogeneous Neumann conditions on the lateral boundaries, we can extend $\phi$ periodically by even reflection on a domain $\Omega$, obtained from $\Pi_{i j}$ by mirror reflections along lateral boundaries, see top domain on figure 2.4. Further, it suffices to show the estimate $(2.22)$ for $\phi$ that solves

$$
\begin{aligned}
\nabla \cdot|\nabla \phi|^{p-2} \nabla \phi & =0, \quad \text { for }(x, y) \in \Pi_{i j} \\
\phi(x, y) & =1 / 2, \quad(x, y) \in \text { top part of } \partial \Omega_{0} \\
\phi(x, y) & =-1 / 2, \quad(x, y) \in \text { bottom part of } \partial \Omega_{0}
\end{aligned}
$$

on a family of smooth periodic domains $\Omega_{0}$ that approximate the periodization of $\Pi_{i j}$ arbitrarily well. An example of $\Omega_{0}$ is depicted at the bottom of figure 2.4. Once 


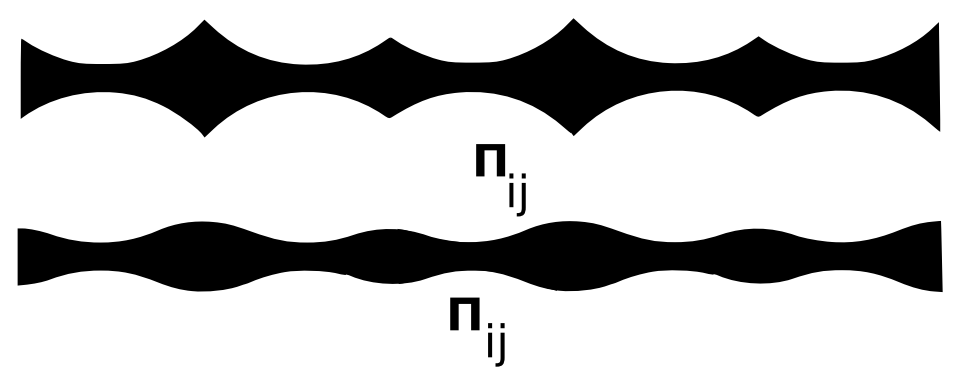

FIG. 2.4. (Top) $\Omega$ Periodization of the neck $\Pi_{i j}$, and (bottom) $\Omega_{0}$ smoothening of the boundary.

the boundary of the domain is smooth, we can use classical elliptic regularity theory as follows. On $\Omega_{0}$ by [21] the solution $\phi$ is $C^{1, \alpha}\left(\bar{\Omega}_{0}\right)$. By the maximum principle for $\phi$ we obtain $|\nabla \phi|>0$. Since $\phi$ is periodic, it could be thought as a solution of $\nabla \cdot|\nabla \phi|^{p-2} \nabla \phi=0$ on a compact domain. Thus $|\nabla \phi| \geq C>0$. Therefore the problem (2.24) is nondegenerate elliptic, and $\phi$ is $C^{\infty}\left(\bar{\Omega}_{0}\right)$ (see e.g. [22] Chapter 3).

We conclude that on a domain $\bar{\Pi}_{i j}$ (see figure 2.5 ) there is $\phi \in C^{\infty}\left(\bar{\Pi}_{i j}\right)$ that solves

$$
\begin{aligned}
\nabla \cdot|\nabla \phi|^{p-2} \nabla \phi & =0, \quad \text { for }(x, y) \in \bar{\Pi}_{i j}, \\
\left.n \cdot \nabla \phi(x, y)\right|_{\partial \bar{\Pi}_{i j}^{ \pm}} & =0, \\
\phi(x, y) & =1 / 2, \quad(x, y) \in \partial \bar{\Pi}_{i j}^{\text {top }}, \\
\phi(x, y) & =-1 / 2, \quad(x, y) \in \partial \bar{\Pi}_{i j}^{\text {bottom }},
\end{aligned}
$$

and it suffices to show that (2.23) holds for such $\phi$, where the constant $C$ is independent of $\delta_{i j}$ and smoothening of the boundary.

Following e.g. [14] we will now apply a Bernstein-type argument. Let

$$
U(x, y)=f(x)|\nabla \phi|^{2}+\lambda \phi^{2},
$$

where we will chose later the constant $\lambda$ and the function $f(x)$. Note that the latter is a function of the horizontal variable $x$ only. Denote

$$
a_{i j}=\delta_{i j}|\nabla \phi|^{p-2}+(p-2)|\nabla \phi|^{p-4} \nabla_{i} \phi \nabla_{j} \phi, i, j=1,2 .
$$

Observe that

$$
\sum_{i, j=1,2} \nabla_{i}\left(a_{i j} \nabla_{j} \phi^{2}\right)=2(p-1)|\nabla \phi|^{p}
$$

because

$$
\begin{aligned}
& \sum_{i, j=1,2} \nabla_{i}\left(|\nabla \phi|^{p-2} \nabla_{i} \phi^{2}+(p-2)|\nabla \phi|^{p-4} \nabla_{i} \phi \nabla_{j} \phi \nabla_{j} \phi^{2}\right) \\
= & 2 \sum_{i, j=1,2} \nabla_{i}\left(\phi|\nabla \phi|^{p-2} \nabla_{i} \phi+(p-2) \phi|\nabla \phi|^{p-4} \nabla_{i} \phi \nabla_{j} \phi \nabla_{j} \phi\right)
\end{aligned}
$$




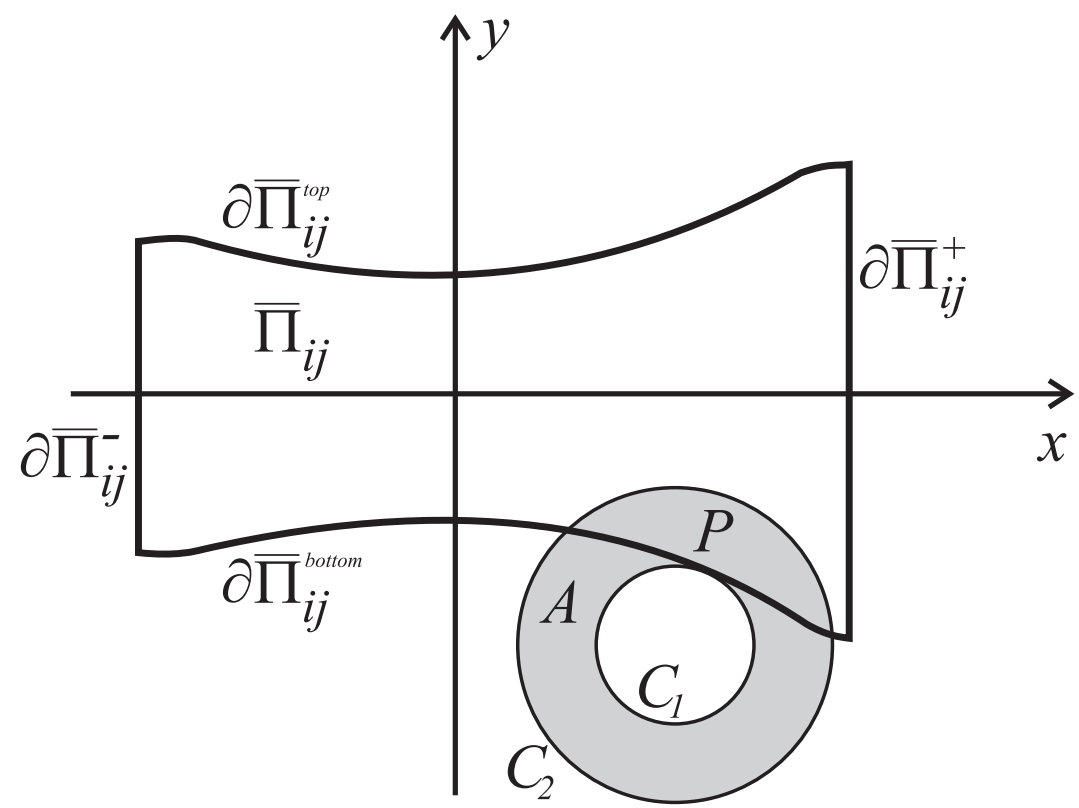

FIG. 2.5. Neck $\bar{\Pi}_{i j}$ with smoothened boundary, and an annulus $A$.

$$
=2(p-1) \sum_{i=1,2} \nabla_{i}\left(\phi|\nabla \phi|^{p-2} \nabla_{i} \phi\right)=2(p-1)|\nabla \phi|^{p} .
$$

Let us show that

$$
\begin{aligned}
\sum_{i, j=1,2} \nabla_{i}\left(a_{i j} \nabla_{j}|\nabla \phi|^{2}\right) & =2|\nabla \phi|^{p-2}\left|\nabla^{2} \phi\right|^{2}+\left.\left.\frac{p-2}{2}|\nabla \phi|^{p-4}|\nabla| \nabla \phi\right|^{2}\right|^{2} \\
& \geq|\nabla \phi|^{p-2}\left|\nabla^{2} \phi\right|^{2}
\end{aligned}
$$

where

$$
\left|\nabla^{2} \phi\right|^{2}=\sum_{i, j=1,2}\left(\nabla_{i} \nabla_{k} \phi\right)^{2}
$$

Differentiate

$$
\nabla \cdot\left(|\nabla \phi|^{p-2} \nabla \phi\right)=0
$$

with respect to $\nabla_{k}$ to obtain

$$
\sum_{i, j=1,2} \nabla_{i}\left(|\nabla \phi|^{p-2} \nabla_{i} \nabla_{k} \phi+(p-2)|\nabla \phi|^{p-4} \nabla_{i} \phi \nabla_{j} \phi \nabla_{j} \nabla_{k} \phi\right)=0 .
$$

Multiply the above by $\nabla_{k} \phi$ and obtain

$$
\sum_{i, j=1,2} \nabla_{k} \phi \nabla_{i}\left(|\nabla \phi|^{p-2} \nabla_{i} \nabla_{k} \phi+(p-2)|\nabla \phi|^{p-4} \nabla_{i} \phi \nabla_{j} \phi \nabla_{j} \nabla_{k} \phi\right)=0 .
$$


It implies that

$$
\begin{aligned}
& \sum_{i, j=1,2} \nabla_{i}\left(|\nabla \phi|^{p-2} \nabla_{i}\left|\nabla_{k} \phi\right|^{2}+(p-2)|\nabla \phi|^{p-4} \nabla_{i} \phi \nabla_{j} \phi \nabla_{j}\left|\nabla_{k} \phi\right|^{2}\right) \\
= & 2 \sum_{i, j=1,2} \nabla_{i} \nabla_{k} \phi\left(|\nabla \phi|^{p-2} \nabla_{i} \nabla_{k} \phi+(p-2)|\nabla \phi|^{p-4} \nabla_{i} \phi \nabla_{j} \phi \nabla_{j} \nabla_{k} \phi\right) .
\end{aligned}
$$

Sum over $k$ to obtain

$$
\begin{aligned}
& \sum_{i, j=1,2} \nabla_{i}\left(|\nabla \phi|^{p-2} \nabla_{i}|\nabla \phi|^{2}+(p-2)|\nabla \phi|^{p-4} \nabla_{i} \phi \nabla_{j} \phi \nabla_{j}|\nabla \phi|^{2}\right) \\
= & 2|\nabla \phi|^{p-2}\left|\nabla^{2} \phi\right|^{2}+\left.\left.\frac{p-2}{2}|\nabla \phi|^{p-4}|\nabla| \nabla \phi\right|^{2}\right|^{2},
\end{aligned}
$$

which is $(2.27)$.

Combining (2.26) and (2.27) for $U=f(x)|\nabla \phi|^{2}+\lambda \phi^{2}$ we obtain

$$
\begin{aligned}
& \sum_{i, j=1,2} \nabla_{i}\left(a_{i j} \nabla_{j} U\right) \\
= & |\nabla \phi|^{2} \sum_{i, j=1,2} \nabla_{i}\left(a_{i j} \nabla_{j} f\right)+f \sum_{i, j=1,2} \nabla_{i}\left(a_{i j} \nabla_{j}|\nabla \phi|^{2}\right) \\
& +2 \sum_{j=1,2} a_{1 j} \nabla_{j}|\nabla \phi|^{2} f^{\prime}(x)+2(p-1) \lambda|\nabla \phi|^{p} \geq 2 f(x)|\nabla \phi|^{p-2}\left|\nabla^{2} \phi\right|^{2} \\
& +2(p-1) \lambda|\nabla \phi|^{p}-C\left|f^{\prime}(x)\right||\nabla \phi|^{p-1}\left|\nabla^{2} \phi\right|-C\left|f^{\prime \prime}(x)\right||\nabla \phi|^{p} \\
\geq & \left(\sqrt{f(x)(p-1) \lambda}-C\left|f^{\prime}(x)\right|\right)|\nabla \phi|^{p-1}\left|\nabla^{2} \phi\right|+\left((p-1) \lambda-C\left|f^{\prime \prime}(x)\right|\right)|\nabla \phi|^{p},
\end{aligned}
$$

where we used the Cauchy-Schwartz inequality in the last step.

We are now ready to choose $f(x)$ and $\lambda$. Recall that in our notation the distance between $\partial B_{i}$ and $\partial B_{j}$ is the smallest at $x=0$ and it equals $\delta_{i j}$ there; see figure 1.3. Noting that $R / 6 \leq \beta_{i j} / 2$, we set $f(x) \equiv 0$, for $|x| \leq R / 12, f(x)=(|x|-R / 12)^{4}$ for $R / 12 \leq|x| \leq R / 6$. We can further define $f(x)$ to be smooth, $R^{4} / 12^{4} \leq f(x) \leq 1$ for $|x| \geq R / 6$, and $f(x) \equiv 1$ near $(x, y) \in \partial \Pi_{i j}^{ \pm}$. Then we can choose a large universal constant $\lambda$ so that

$$
\left(\sqrt{f(x)(p-1) \lambda}-C\left|f^{\prime}(x)\right|\right)|\nabla \phi|^{p-1}\left|\nabla^{2} \phi\right|+\left((p-1) \lambda-C\left|f^{\prime \prime}(x)\right|\right)|\nabla \phi|^{p}>0 .
$$

Therefore $U(x, y)=f(x)|\nabla \phi(x, y)|^{2}+\lambda \phi^{2}(x, y)$ satisfies the maximum principle. Since $U$ satisfies the maximum principle on $\Omega_{0}$ (see figure 2.4) as well, its maximum is achieved on the top or the bottom boundaries of $\bar{\Pi}_{i j}$. Using $-1 / 2 \leq \phi \leq 1 / 2$, we obtain

$$
\max _{(x, y) \in \partial \bar{\Pi}_{i j}^{ \pm}}|\nabla \phi(x, y)|^{2} \leq \max _{(x, y) \in \bar{\Pi}_{i j}} U(x, y) \leq C+\max _{(x, y) \in \partial \bar{\Pi}_{i j}^{\text {top }}, \partial \bar{\Pi}_{i j}^{\text {bottom }}} f(x)|\nabla \phi(x, y)|^{2} .
$$

Hence we only need to obtain estimates for $f(x)|\nabla \phi(x, y)|^{2}$ on the top and the bottom boundaries. We will obtain them by using barriers for $\nabla \phi(x, y)$. Since $f(x) \equiv 0$, for $|x| \leq R / 12$, we need to estimate $\nabla \phi(x, y)$, for $R / 12 \leq|x| \leq R$ only. Fix any point $P:(x, y) \in \partial \bar{\Pi}_{i j}^{\text {bottom }}$, such that $R / 12 \leq|x| \leq R$. Consider two concentric circles $C_{1}$ 
and $C_{2}$ of radii $R / 600$ and $R / 1200$, respectively, such that its interior circle is tangent to the $\partial \bar{\Pi}_{i j}^{\text {bottom }}$ at the point $P$ and the center of the circles does not lie in $\bar{\Pi}_{i j}$ (see figure 2.5). Note that the annulus $A$ between these two circles does not intersect the top boundary $\partial \bar{\Pi}_{i j}^{\text {top }}$ for any choice of $P:(x, y) \in \partial \bar{\Pi}_{i j}^{\text {bottom }}$ with $R / 12 \leq|x| \leq R$. Therefore the function $\psi$, the solution of

$$
\begin{aligned}
\nabla \cdot|\nabla \psi|^{p-2} \nabla \psi & =0, \quad \text { for }(x, y) \in A, \\
\psi(x, y) & =-1 / 2, \quad(x, y) \in C_{1}, \\
\psi(x, y) & =1 / 2, \quad(x, y) \in C_{2},
\end{aligned}
$$

will be a super-solution for $\phi$ in $A \cap \bar{\Pi}_{i j}$. Lastly $\psi(x, y)=\phi(x, y)=-1 / 2$ at $P$, and thus $\psi$ is a barrier for $\phi$ at the point $P$. We have $|\nabla \psi(x, y)| \leq C, C=C(R)$, and therefore we obtain

$$
\max _{(x, y) \in \partial \bar{\Pi}_{i j}^{\text {bottom }}} f(x)|\nabla \phi(x, y)|^{2} \leq C, C=C(R) .
$$

Using the last inequality and a similar inequality at the top boundary in (2.28), we obtain (2.23).

\section{Proofs of Lemma $\mathbf{1 . 2}$ and Theorem $\mathbf{1 . 3}$}

We recall, that for a pair of vertices $\mathrm{x}_{i}$ and $\mathrm{x}_{j}$, connected by the edge $\Pi_{i j}$, the interparticle distance is the distance between the corresponding inclusions

$$
\delta_{i j}=\left|\mathrm{x}_{i}-\mathrm{x}_{j}\right|-2 R .
$$

Similarly, the interparticle distance between an inclusion and one of the special (boundary) vertices $\mathrm{x}_{N+1}$ and $\mathrm{x}_{N+2}$ is set as the distance between the inclusion and the boundary. When concentration of inclusions is high, we expect that a typical $\delta_{i j} \ll 1$, and, therefore, we are interested in asymptotic values of $g_{i j}$ as $\delta_{i j} \rightarrow 0$.

We will prove a slightly stronger version of Lemma 1.2. More specifically, we have the following result.

Lemma 3.1. Consider a neck $\Pi_{i j}$ between two inclusions $B_{i}$ and $B_{j}$. Suppose $g_{i j}$ is given by (2.12). As $\delta_{i j} \rightarrow 0$

$$
g_{i j}=g_{i j}^{o}+O\left(f\left(\delta_{i j}\right)\right),
$$

where the constants $g_{i j}^{o}=\mathbf{G}\left(\delta_{i j}\right)$ are given in table (1.11), and

$$
f(\delta)=\left\{\begin{array}{l}
1, \text { if } p=2 \\
\ln \delta, \text { if } p=3, d=3 \\
\delta^{-p+(d+3) / 2}, \text { otherwise }
\end{array}\right.
$$

where $p \geq 2$ is the power of the power-law nonlinearity and the dimension $d=2$ or $d=3$. Consider a neck $\Pi_{i j}$ between an inclusion $B_{i}$ and the flat boundary $j=N$ or $j=N+1$. As $\delta_{i j} \rightarrow 0$

$$
g_{i j}=g_{i j}^{o}+O\left(f\left(\delta_{i j}\right)\right) \text {, }
$$

where $g_{i j}^{o}$ is given by

$$
g_{i j}^{o}=2^{p-1} \mathbf{G}\left(2 \delta_{i j}\right) .
$$


Proof. Consider a neck $\Pi_{i j}$ between two inclusions $B_{i}$ and $B_{j}$. For any $\phi \in V_{\Pi_{i j}}$ and $\boldsymbol{u} \in V_{\Pi_{i j}}^{*}$ integrating by parts $\phi \nabla \cdot|\boldsymbol{u}|^{p-2} \boldsymbol{u}=0$, we obtain the following identity:

$$
\frac{1}{2} \int_{\partial B_{i} \cup \partial B_{j}}|\boldsymbol{u}|^{p-2} \boldsymbol{n} \cdot \boldsymbol{u} d s=\int_{\Pi_{i j}}|\boldsymbol{u}|^{p-2} \nabla \phi \cdot \boldsymbol{u} d x d y .
$$

By the direct (2.12) and the dual (2.16) variational principles we have

$$
\left[\frac{p}{2} \int_{\partial B_{i} \cup \partial B_{j}}|\boldsymbol{u}|^{p-2} \boldsymbol{n} \cdot \boldsymbol{u} d s-(p-1) \int_{\Pi_{i j}}|\boldsymbol{u}|^{p} d x d y\right] \leq g_{i j} \leq \int_{\Pi_{i j}}|\nabla \phi|^{p} d x d y .
$$

Using identity (3.5) in the last inequality we obtain the following variational bounds: for any $\phi \in V_{\Pi_{i j}}$ and $\boldsymbol{u} \in V_{\Pi_{i j}}^{*}$

$$
\int_{\Pi_{i j}}\left(p|\boldsymbol{u}|^{p-2} \nabla \phi \cdot \boldsymbol{u}-(p-1)|\boldsymbol{u}|^{p}\right) d x d y \leq g_{i j} \leq \int_{\Pi_{i j}}|\nabla \phi|^{p} d x d y .
$$

Evaluating (3.6) with

$$
\phi=\frac{y}{H(x)}, \boldsymbol{u}=\left(0, \frac{1}{H(x)}\right)
$$

we obtain $g_{i j}^{l} \leq g_{i j} \leq g_{i j}^{u}$ for

$$
g_{i j}^{l}=\int_{\Pi_{i j}} \frac{1}{[H(x)]^{p}} d x d y, g_{i j}^{u}=\int_{\Pi_{i j}}\left[\frac{\left[y H^{\prime}(x)\right]^{2}}{[H(x)]^{4}}+\frac{1}{[H(x)]^{2}}\right]^{p / 2} d x d y .
$$

It suffices to show that

$$
\begin{aligned}
\left|g_{i j}^{u}-g_{i j}^{l}\right| & =\int_{\Pi_{i j}}\left(\left[\frac{\left[y H^{\prime}(x)\right]^{2}}{[H(x)]^{4}}+\frac{1}{[H(x)]^{2}}\right]^{p / 2}-\frac{1}{[H(x)]^{p}}\right) d \boldsymbol{x} \\
& \leq \int_{\Pi_{i j}} \frac{\left[y H^{\prime}(x)\right]^{2}}{[H(x)]^{p+2}} d \boldsymbol{x}=O\left(f\left(\delta_{i j}\right)\right)
\end{aligned}
$$

and

$$
g_{i j}^{l}=\int_{\Pi_{i j}} \frac{1}{[H(x)]^{p}} d \boldsymbol{x}=g_{i j}^{o}+O\left(f\left(\delta_{i j}\right)\right),
$$

where $g_{i j}^{u}$ and $g_{i j}^{l}$ are defined by (3.8). Using Lemma 2.3 we know that as $\delta_{i j} \rightarrow 0$, the width of $\Pi_{i j}$ stays bounded away from zero and the line segment $\mathrm{x}_{i} \mathrm{x}_{j}$ that connects the centers of inclusions always crosses $\Pi_{i j}$. We then verify (3.9) and (3.10) by lengthy, but straightforward computations.

The argument for the boundary necks is the same, whereas the last estimate (3.4) is obtained by observing that two identical boundary necks of the height $\delta_{i j}$ glued along the flat horizontal boundary give one neck between two inclusions, but of height $2 \delta_{i j}$.

Proof. [Proof of Theorem 1.3] Denoting

$$
g(\delta)=\min _{\Pi_{i j}} g_{i j},
$$


and using (1.8) and (1.9) we obtain

$$
\hat{a}_{d} \leq \hat{a} \leq \min _{t_{i}} \sum_{\Pi_{i j}}(1+C / g(\delta)) g_{i j}\left|t_{i}-t_{j}\right|^{p}=(1+C / g(\delta)) \hat{a}_{d},
$$

where the constant $C$ depends on $R$ and $L$ only. It implies that

$$
\frac{\left|\hat{a}-\hat{a}_{d}\right|}{\hat{a}_{d}} \leq \frac{C}{g(\delta)} \equiv \frac{C}{\min _{\Pi_{i j}} g_{i j}} .
$$

Theorem 1.3 follows, when we evaluate the right-hand side of the last inequality using the table (1.11).

\section{Proof of Theorem 1.6}

In order to avoid considering four different cases we will assume that $p \geq 3$.

Proof. We use the notation

$$
\begin{aligned}
& \hat{a}_{d}=\min _{t_{i}, i=1, \ldots, N} I\left(t_{1}, \ldots, t_{N}\right), I\left(t_{1}, \ldots, t_{N}\right)=\sum_{\Pi_{i j}} g_{i j}\left|t_{i}-t_{j}\right|^{p} \\
& \hat{a}_{d}^{o}=\min _{t_{i}, i=1, \ldots, N} I^{o}\left(t_{1}, \ldots, t_{N}\right), I^{o}\left(t_{1}, \ldots, t_{N}\right)=\sum_{\Pi_{i j}} g_{i j}^{o}\left|t_{i}-t_{j}\right|^{p} .
\end{aligned}
$$

We have (see e.g. [6]) a discrete maximum principle: if all $g_{i j}>0$ (or all $g_{i j}^{o}>0$ ), then there exists a unique $\left\{t_{1}, t_{2}, \ldots t_{N}\right\}$ that solve the minimization problem (4.1) or (4.2), with the estimate

$$
-1 \leq t_{k} \leq 1,
$$

for any $t_{k}$. By Lemma 2.2

$$
\hat{a}_{d} \leq \hat{a} \leq \min _{t_{i}, i=1, \ldots, N} \sum_{\Pi_{i j}}\left(g_{i j}+C\right)\left|t_{i}-t_{j}\right|^{p},
$$

with $t_{N+1}=1, t_{N+2}=-1$, and the constant $C$ in (4.3) depends on $R$ and $L$ only. Using $-1 \leq t_{k} \leq 1$ inequalities (4.3) become

$$
\hat{a}_{d} \leq \hat{a} \leq \hat{a}_{d}+C .
$$

Existence of the percolating cluster and (1.11) implies that

$$
\hat{a}_{d} \geq C \delta^{-p+(d+1) / 2}, \hat{a}_{d}^{o} \geq C \delta^{-p+(d+1) / 2} .
$$

Thus

$$
\frac{\left|\hat{a}-\hat{a}_{d}\right|}{\hat{a}_{d}} \leq C \delta^{p-(d+1) / 2},
$$

and it means that Theorem 1.3 holds if there is a $\delta$-percolating cluster. The proof of Corollary 1.4 requires more work, mainly because in contrast to the case considered in $[6]$ the quantity

$$
\left|g_{i j}-g_{i j}^{o}\right| \rightarrow \infty, \text { as } \delta \rightarrow 0, \text { for } \Pi_{i j} \in \mathbb{D}_{\delta},
$$


where we denote by $\mathbb{D}_{\delta}$ the set of $\delta$-small necks: $\mathbb{D}_{\delta}=\left\{\Pi_{i j} \mid \delta_{i j} \leq \delta\right\}$. We also denote

$$
g^{o}(\delta)=\min _{\Pi_{i j} \in \mathbb{D}_{\delta}} g_{i j}^{o}
$$

From (3.9), (3.10), and (1.11) we estimate

$$
\left|g_{i j}-g_{i j}^{o}\right| \leq C \delta^{-p+(d+3) / 2} \text {, if } \Pi_{i j} \notin \mathbb{D}_{\delta} .
$$

It means that

$$
(1-C \delta) g_{i j}^{o} \leq g_{i j} \leq(1+C \delta) g_{i j}^{o}, \text { if } \Pi_{i j} \in \mathbb{D}_{\delta} .
$$

Hence for any $\left\{t_{1}, t_{2}, \ldots t_{N}\right\}$, that satisfy $-1 \leq t_{k} \leq 1$, we obtain

$$
\begin{array}{r}
(1-C \delta) I^{o}\left(t_{1}, \ldots, t_{N}\right)-C \delta^{-p+(d+3) / 2} \leq I\left(t_{1}, \ldots, t_{N}\right) \\
\leq(1+C \delta) I^{o}\left(t_{1}, \ldots, t_{N}\right)+C \delta^{-p+(d+3) / 2} .
\end{array}
$$

It implies that

$$
(1-C \delta) \hat{a}_{d}^{o}-C \delta^{-p+(d+3) / 2} \leq \hat{a}_{d} \leq(1+C \delta) \hat{a}_{d}^{o}+C \delta^{-p+(d+3) / 2} .
$$

Using the last inequality, estimates (4.6), and (4.5) we obtain (1.14).

Acknowledgments. This work was supported by grants BSF-2005133, DMS0604600, DMS-0619587, DMS-0908011.

\section{REFERENCES}

[1] V. Ambegaokar, B.I. Halperin and J.S. Langer, Hopping conductivity in disordered systems, Phys. Rev. B, 4(8), 2612-2620, 1971.

[2] L. Berlyand, L. Borcea and A. Panchenko, Network approximation for effective viscosity of concentrated suspensions with complex geometry, SIAM J. Math. Anal., 36(5), 1580-1628, 2005 .

[3] L. Berlyand, Yu. Gorb and A. Novikov, Discrete network approximation for highly-packed composites with irregular geometry in three dimensions, Uppsala Proceedings in Multiscale Methods in Science and Engineering, Springer Lecture Notes, 21-58, 2005.

[4] L. Berlyand, Yu. Gorb and A. Novikov, Fictitious fluid approach and anomalous blow-up of the dissipation rate in a $2 D$ model of concentrated suspensions, Arch. Rat. Math. Anal, 193(3), 585-622, 2009.

[5] L. Berlyand and A. Kolpakov, Network approximation in the limit of small interparticle distance of the effective properties of a high contrast random dispersed composite, Arch. Rat. Math. Anal., 159(3), 179-227, 2001.

[6] L.V. Berlyand and A. Novikov, Error of the network approximation for densely packed composites with irregular geometry, SIAM J. Math. Anal., 34(2), 385-408, 2002.

[7] L. Borcea, Asymptotic analysis of quasistatic transport in high contrast conductive media, SIAM J. Appl. Math., 59(2), 597-639, 1999.

[8] L. Borcea, J.G. Berryman and G. Papanicolaou, Matching pursuit for imaging high contrast conductivity, Inverse Problems, 15(4), 811-849, 1999.

[9] L. Borcea and G. Papanicolaou, Network approximation for transport properties of high contrast materials, SIAM J. Appl. Math., 58(2), 501-539, 1998.

[10] L. Borcea and G. Papanicolaou, Low Frequency Electromagnetic Fields in High Contrast Media, Surveys on Solution Methods for Inverse Problems, D. Colton, H. W. Engl, A. Louis, J. R. McLaughlin and W. Rundell (eds), Springer Vienna/New York, 2000.

[11] L. Borcea, J.G. Berryman and G.C. Papanicolaou, High-contrast impedance tomography, Inverse Problems, 12(6), 835-858, 1996.

[12] A. Cherkaev, Variational Methods for Structural Optimization, Applied Mathetmatical Sciences, Springer-Verlag, New York, 140, 2000. 
[13] J.P. Clerc, G. Giraud, J.M. Laugier and J.M. Luck, The electrical conductivity of binary disordered systems, percolation clusters, fractals and related models, Advances in Physics, 39(3), 1910-309, 1990.

[14] L.C. Evans and W. Gangbo, Differential equations methods for the Monge-Kantorovich mass transfer problem, Mem. Amer. Math. Soc., 137, 653, 1999.

[15] H. Federer, Geometric Measure Theory, Springer, Berlin, 1969.

[16] B.I. Halperin, Remarks on percolation and transport in networks with a wide range of bond strengths, Physica D, 38, 179-183, 1989.

[17] V.V. Jikov, S.M. Kozlov and O.A. Oleinik, Homogenization of Differential Operators and Integral Functionals, Springer-Verlag: Berlin, Heidelberg, 1994.

[18] J.B. Keller, Conductivity of a medium containing a dense array of perfectly conducting spheres or cylinders or nonconducting cylinders, J. Appl. Phys., 34(4), 991-993, 1963.

[19] J. Koplik, Creeping flow in two-dimensional networks, J. Fluid Mech., 119, 219-247, 1982.

[20] S.M. Kozlov, Geometrical aspects of averaging, Russ. Math. Surveys, 44(22), 91-144, 1989.

[21] G.M. Lieberman, Boundary regularity for solutions of degenerate elliptic equations, Nonlinear Anal., 11, 1203-1219, 1988

[22] O.A. Ladyzhenskaya, Ural'tseva and N. Nina, Linear and Quasilinear Elliptic Equations, Academic Press, New York-London, 1968

[23] R. McPhedran, Transport property of cylinder pairs of the square array of cylinders, Proc. R. Soc. London. A, 408, 31-43, 1986.

[24] R. McPhedran, L. Poladian and G.W. Milton, Asymptotic studies of closely spaced, highly conducting cylinders, Proc. R. Soc. Lond. A, 415, 185-196, 1988.

[25] G.W. Milton, The Theory of Composites, Cambridge Monographs on Applied and Computational Mathematics, Cambridge University Press, Cambridge, 6, 2002.

[26] A. Novikov and G. Papanicolaou, Eddy viscosity of cellular flows, J. Fluid Mech., 446, 173-198, 2001.

[27] A. Novikov, G. Papanicolaou and L. Ryzhik, Boundary layers for cellular flows at high Péclet numbers, Commun. Pure Appl. Math., 58, 7, 867-922, 2005.

[28] C.B. Shah and Y.C. Yortsos, The permeability of strongly-disordered systems, Physics of Fluids, 8(1), 280-282, 1996. 